\title{
The 4th Palermo Swift-BAT catalogue: 100 months of survey of the hard X-ray sky.
}

\author{
A. Segreto*, G. Cusumano, V. La Parola and A. Maselli \\ INAF, Istituto di Astrofisica Spaziale e Fisica Cosmica di Palermo, Via U. La Malfa 153, I-90146 \\ Palermo, Italy \\ E-mail: segretodifc.inaf.it, cusumanodifc.inaf.it, \\ laparola@ifc.inaf.it and masellidifc.inaf.it
}

The 4th Palermo Swift-BAT hard X-ray catalogue is obtained by analyzing data acquired in the first 9 years of the Swift mission. The survey covers $50 \%$ of the sky to a $15-150 \mathrm{keV}$ flux limit of $0.27 \mathrm{mCrab}\left(\sim 5.4 \times 10^{-12} \mathrm{erg} \mathrm{cm}^{-2} \mathrm{~s}^{-1}\right)$. We use a source detection algorithm that optimizes the energy band and the time interval achieving the maximum signal to noise ratio for each pixel of the all-sky map. We obtain a list of 1710 source candidates with a negligible number of spurious detections. The identification of the source counterparts in the softer energy band is pursued through the analysis of soft X-ray field observations or a cross-correlation with source databases. The 4th Palermo Swift-BAT catalogue consists of $\sim 19 \%$ Galactic sources, $\sim 57 \%$ extragalactic sources, $\sim 13 \%$ sources with a counterpart at softer energies whose nature has not been determined yet while $\sim 11 \%$ still lack any associated counterpart.

Swift: 10 Years of Discovery,

2-5 December 2014

La Sapienza University, Rome, Italy

\footnotetext{
${ }^{*}$ Speaker.
} 


\section{Introduction}

The Burst Alert Telescope (BAT; [1]) onboard the Swift observatory [2] is performing since November 2004 a continuous monitoring of the entire hard X-ray sky, collecting imaging, timing and spectral information.

BAT is a coded-aperture imaging camera operating in the $15-150 \mathrm{keV}$ energy range, with a large field of view (FoV) of 1.4 steradian (half coded) and a point spread function of 17 arcmin FWHM (Full Width Half Maximum). BAT operates as a hard X-ray monitor with the main goal of catching gamma ray bursts and fast transient phenomena (with timescales up to several minutes), locating onboard their position with an accuracy of 1 to 4 arc-minutes within several seconds. While it is hunting for gamma-ray bursts, it also records the flux variability of known X-ray sources and discovers many new X-ray transients.

Thanks to the good sensitivity, the large FoV and the pointing strategy, that provide a coverage of a fraction of between $50 \%$ and $80 \%$ of the sky every day, BAT has allowed an extraordinary improvement of our knowledge of the sky in the hard X-ray energy range.

Using a dedicated software [3] we are producing the Palermo Swift-BAT Catalogue series (39month [4], 54-month, [5], 66-month[6]). In this paper, we describe the Fourth Palermo Swift-BAT hard X-ray catalogue obtained from the analysis of the data relevant to the first 100 months of the Swift mission (December 2004 - March 2013).

\section{Global survey properties}

After $\sim 100$ months of all-sky survey monitoring, BAT limiting flux has reached a few $10^{-12}$ erg $\mathrm{cm}^{-2} \mathrm{~s}^{-1}$, depending on the sky direction. In Fig. 1 we show the $5 \sigma$ limiting flux of the BAT survey as a function of the exposure time.

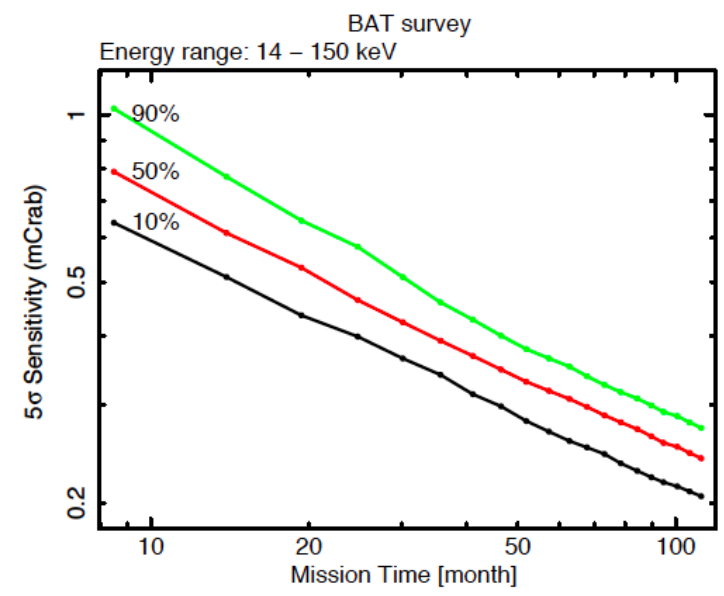

Figure 1: Sensitivity of the BAT survey as a function of exposure time. The 3 different lines show the fraction of the all-sky area that is sensitive to a given flux limit. 
In Fig. 2 we show the fraction of the sky as a function of the detection limiting flux in the 15-150 keV skymap for Galactic and extragalactic regions.

\section{Methodology}

The survey data were analyzed by applying an analysis method that allows to optimize the source detection for variable sources: the data are collected in nine energy bands and binned in 15-day time intervals, and after building several all-sky maps in contiguous energy bands and time intervals we apply a blind search for significance excesses above an appropriate detection threshold. As the presence of bright sources (e.g. in the Galactic center) may hide the presence of close fainter sources, the above procedure is repeated on the all-sky maps obtained after subtracting from the data the illumination pattern produced by all the sources detected in the first search.

\section{Association strategy}

Since the source localization improves with the $\mathrm{S} / \mathrm{N}$ (while the error radius associated to it becomes smaller), for all the sources we have optimized their position deriving it from the best significance map.

In order to identify the nature of the detected sources, we have searched for possible counterparts in other energy bands. Our main strategy consists in using soft X-ray observations whose field of view covers the position of the BAT sources, and in searching sources inside the BAT error box. We have used mainly Swift-XRT follow-up observations (most of which were requested by us) or archival observations of previous soft X-ray satellites. The identification of the soft X-ray counterparts was pursued using the much smaller soft X-ray error box to search on the SIMBAD and NED archives.

The BAT sources without a counterpart in soft X-ray field observations were cross-correlated with a list of possible counterparts obtained merging appropriate catalogues (high and low mass X-

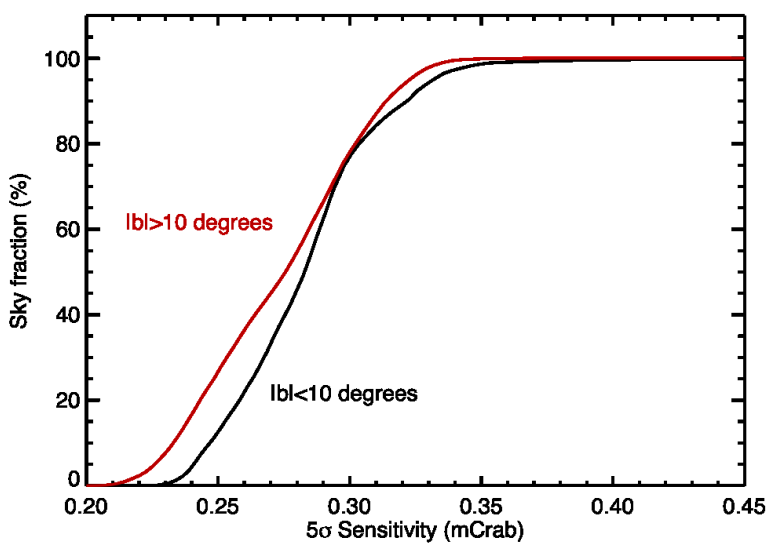

Figure 2: Survey sky coverage for a detection threshold of $5 \mathrm{~s}$ after 100 months of survey, on and off the Galactic plane. 


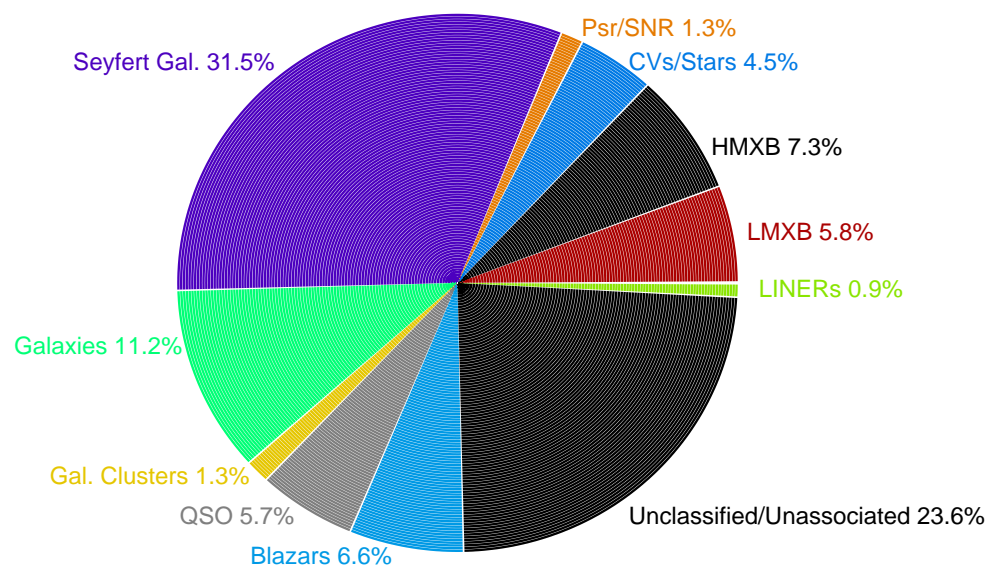

Figure 3: Detailed distribution of the catalogue sources among different classes of cosmic sources.

ray binaries, cataclysmic variables, supernova remnants and pulsars, Seyfert galaxies, unclassified AGNs, interacting galaxies, LINERs, and $\gamma$-ray sources from the SIMBAD database as of January 2010; the Roma-BZCAT [7]; the ROSAT All-Sky Survey (RASS) Bright Sources Catalogue [8]).

After the application of the association procedures, $\sim 90 \%$ of BAT sources have an associated counterpart; $\sim 57 \%$ of them are extragalactic, $\sim 19 \%$ are Galactic, $\sim 13 \%$ are soft X-ray emitters whose nature has not been determined yet, while $\sim 11 \%$ still lack any associated counterpart. The detailed distribution among different classes of cosmic sources is shown in Figure 3.

The on-line version of the 4th Palermo Swift-BAT catalog is available at the following address: http://bat.ifc.inaf.it/100m_bat_catalog/100m_bat_catalog_v0.0.htm

\section{References}

[1] S. D. Barthelmy, et al., The Burst Alert Telescope (BAT) on the SWIFT Midex Mission, Space Science Reviews, 120 (2005), 1435

[2] N. Gehrels, et al., The Swift gamma-ray burst mission, ApJ, 611 (2004), 1005

[3] A. Segreto, et al. The Palermo Swift-BAT hard X-ray catalogue. I. Methodology, A\&A, 510 (2010), A47

[4] G. Cusumano, et al., The Palermo Swift-BAT hard X-ray catalogue. II. Results after 39 months of sky survey, $A \& A, \mathbf{5 1 0}$ (2010), A48

[5] G. Cusumano, et al., The Palermo Swift-BAT hard X-ray catalogue. III. Results after 54 months of sky survey, $A \& A, \mathbf{5 2 4}$ (2010), A64

[6] http://bat.ifc.inaf.it/bat_catalog_web/66m_bat_catalog.html

[7] E. Massaro, et. al, Roma-BZCAT: a multifrequency catalogue of blazars, A\&A, 495 (2009), 691

[8] W. Voges, et al., The ROSAT all-sky survey bright source catalogue, A\&A, 349 (1999), 389 